\title{
ACERCA DE LA INDISOLUBILIDAD DEL MATRIMONIO...
}

\author{
Gloria Claro Montes'
}

Pocos temas son objeto de debate tan virulento y enconado como el divorcio en nuestro país. Algunos consideran que no podemos siquiera empezar a hablar de libertad si es que el estado no reconoce el derecho de las parejas a poner término a su matrimonio. Otros en cambio, creen que la dictación de una ley de divorcio promovería el libertinaje y la llevaría a la decadencia moral de la sociedad, lo que los hace oponerse tenazmente a ella.

Aquellos que se rechazan la idea de legislar sobre el divorcio lo hacen en virtud de varios argumentos de tipo filosófico, constitucional y político. Uno de los principales argumentos de carácter filosófico que se esgrime, es que el matrimonio es indisoluble por naturaleza y que ninguna ley podría legítimamente alterar dicha naturaleza y admitir su disolución. ${ }^{2}$ Desde el punto de vista constitucional se sostiene que la Constitución establece la obligación de proteger la familia y que aprobar una ley que aceptara el divorcio violaría dicho precepto constitucional. Por último, se argumenta desde el punto de vista político que no es conveniente la dictación de una ley de divorcio, ya que de la aceptación de éste se seguiria la destrucción de la familia y la decadencia de la sociedad, agregándose que existe un interés de toda la sociedad -y en particular de las autoridades- de evitar ese desastre.

En nuestra opinión, estos argumentos no son lo suficientemente fuertes para mantener la situación actual, ni para impedir la dictación de una ley que permita el divorcio. Parece enteramente debatible sostener que la indisolubilidad del matrimonio sea una característica natural del matrimonio. Asimismo, no resulta claro que adoptar una ley que favorezca el divorcio viole el precepto constitucional de proteger la familia, o que de una ley de divorcio se siga la destrucción de la familia y menos la de la sociedad.

Desgraciadamente por las limitaciones de este artículo no podremos referimos a todos los argumentos antes expuestos, así que hemos decidido avocarnos al análisis de uno sólo de los argumentos, aquel de carácter filosófico, porque creemos que es probablemente central en la discusión. Sin embargo,

I Abogada. Pontificia Universidad Católica de Chile. Master en Derecho Universidad de Michigan.

2 José Miguel Ibáñez Langlois, "21 Slogans divorcistas", 17-28. Editorial Andrés Bello. 1991; José Joaquín Ugarte G., "La Indisolubilidad del Matrimonio", en Seminario sobre Matrimonio y Familia. Pontificia Universidad Católica de Chile, Facultad de Derecho. 1994. 
antes de adentrarnos de lleno en éste, hemos creído conveniente referirnos a la situación actual, ya que, cualquiera sea la postura que se tenga en relación a una ley de divorcio, es necesario convencernos de que lo que está ocurriendo actualmente en nuestro país es aberrante y nocivo para nuestro sistema social y jurídico y que debe de todas maneras modificarse.

\section{I.- Unas palabras acerca de la actual situación.}

Nuestra ley de matrimonio civil establece que el único matrimonio válido es el contraído de conformidad a las leyes civiles. El código civil dispone que el matrimonio es un contrato indisoluble y, como ya hemos dicho, no reconoce el divorcio vincular. Sin embargo, no obstante las definiciones legales, la realidad es que los matrimonios de los chilenos también fracasan y cuando ello ocurre los cónyuges quieren disolver sus uniones en la manera menos traumática posible y seguir adelante con sus vidas. Como la legislación no da una solución efectiva a sus problemas, lo que se ha hecho en el último tiempo es torcer el espíritu de la ley. Así las cosas en Chile, en la práctica los matrimonios no son indisolubles, ellos se "disuelven" a través del mecanismo de la nulidad del contrato matrimonial.

El uso y abuso de la nulidad afecta nuestro sistema, no solo desde una perspectiva legal sino también social.

Legalmente se institucionaliza una mentira. El uso de la incompetencia del oficial del registro civil como causal de nulidad (que es sin duda la más utilizada) es casi siempre -y uno es generoso diciendo casi siempre- una mentira. Cuando los esposos se están casando, no quieren que su matrimonio sea nulo así que se preocupan de cumplir con los requisitos de solemnidad. Sin embargo, cuando las cosas van mal, los cónyuges empiezan a ver que de qué modo pueden ponerle fin a su matrimonio. Como el divorcio no es posible, la alternativa de obtener la nulidad emerge. Aún cuando los esposos saben que están incurriendo en un proceso fraudulento la falta de otras opciones los deja sin más que recurrir al resquicio de la incompetencia del oficial del registro civil. El problema es que esta opción contamina todo el sistema legal. En efecto, no sólo los esposos saben que es falso el procedimiento en el que están involucrándose. sino que también lo saben los testigos que en él participan, los abogados, y lo que es peor, los jueces que dictan la resolución.

A mi juicio los chilenos tomamos muy frívolamente el daño que por esta vía se infringe a nuestro sistema jurídico. Se permite, sin mayor escándalo, que los ciudadanos se involucren en un procedimiento que es a todas luces una farsa y que lo daña directamente. En efecto, se pierde la credibilidad del sistema legal y judicial para otros procedimientos; se permite sin problema que los abogados bajen sus estándares éticos en este procedimiento, lo que hace que difícilmente podamos exigir una mayor rigurosidad cuando se enfrenten a casos de otra 
naturaleza. Por otra parte el procedimiento de la nulidad deteriora el valor de la prueba testimonial, privándonos de una de las más importantes herramientas de nuestro sistema probatorio, y por si fuera poco, contribuye al desprestigio de los tribunales, quienes se ven obligados a aceptar una mentira patente y a hacer la vista gorda.

Este sistema nos afecta también socialmente.

En primer lugar nos daña porque genera corrupción. Uno de los grandes orgullos de Chile es que no es un país corrupto, pero cuando las partes, los testigos, los abogados y los jueces claramente se involucran en un proceso de fraude a la ley; cuando testigos y abogados avalan una mentira; cuando los afectados mienten abiertamente bajo la obligación de decir verdad. ¿Cómo podremos evitar que el sistema se corrompa?

El mecanismo de la nulidad también nos afecta socialmente porque es objeto de negocio y extorsión. Aunque la nulidad es en principio, un proceso adversarial, en la práctica, los cónyuges necesitan estar de acuerdo para producir la prueba que permita la obtención del resultado deseado. En este contexto, la nulidad debe ser negociada. Lo triste y fuerte del asunto es que los objetos transados son generalmente los hijos del matrimonio, sus visitas y alimentos.

En tercer lugar el sistema perjudica el tejido social porque deja a muchas familias sin reconocimiento legal. Atendido a que el procedimiento de nulidad es caro y a que no todas las parejas se ponen de acuerdo para solicitarla, una alta proporción de matrimonios fracasados permanece legalmente casados. Esto último, sin embargo, no previene a los cónyuges de crear "familias de facto" con otras parejas. El dilema que esto presenta es que estas familias no son reconocidas por la ley, y que los hijos que en ellas se engendran nacen en la ilegitimidad.

El hecho de que exista un doble standard, de que personas que puedan disolver sus matrimonios por la via de la nulidad mientras otros no pueden hacerlo, es un elemento a considerar cuando justificamos la existencia de este sistema. ¿Cómo es posible que algunos ciudadanos tengan acceso a formar segundas familias que sean reconocidas por la ley y otros no?; ¿Qué pasa con los hijos de estas uniones de hecho?; ¿Deben ellos pagar las consecuencias de que sus padres no puedan obtener la disolución legal de matrimonios anteriores y aceptar que sus derechos se vean afectados en varias áreas?

$\mathrm{Al}$ parecer hay un cierto consenso de que la actual situación es dañina para nuestro sistema legal, para nuestra sociedad y que además es injusta. Sin embargo, lo que falta es ponerse de acuerdo en la solución. Algunos creen que el actual sistema es malo porque finalmente admite el divorcio, que es lo malo en sí mismo. En este entendido, la solución iría por ponerle término a la nulidad fraudulenta y cerrar toda posibilidad a un divorcio vincular. ${ }^{3}$ Otros piensan que

\footnotetext{
${ }^{3}$ Id. en $97-98$.
} 
el actual sistema es malo, pero como reconocen la necesidad de dejar un válvula de escape y creen que la alternativa de divorcio es más nociva aún, consideran conveniente mantener la situación tal como está. Un tercer grupo, considera que el divorcio debe ser autorizado porque, al fin y al cabo, se trata del tipo de decisión que debe ser tomada por los individuos y en la cual el estado no puede intervenir, sino sólo regular sus efectos cuando dañan a terceros.

La mayoría de los del primer y segundo grupo, quienes se oponen a un proyecto de ley de divorcio vincular, lo hacen, tal como mencionamos al comienzo de este artículo, en base a argumentos filosóficos, constitucionales y políticos. El argumento filosófico, que nos ocupará por el resto de este trabajo, se basa en que el matrimonio sería una institución indisoluble por naturaleza. Veamos de qué se trata.

\section{II.- Argumento filosófico en contra del divorcio.}

De acuerdo a este argumento, el matrimonio es indisoluble por naturaleza. La indisolubilidad del matrimonio no sería un asunto de convicciones religiosas, sino una de las características naturales de dicha institución. En este contexto. la ley positiva no podría contravenir o violentar la naturaleza de la institución. Una ley que lo hiciera, que como la que autorizara el divorcio, sería contraria al Derecho Natural, injusta, moralmente mala.

Este argumento amerita dos reflexiones. No todas las personas creen que existan caracteristicas que pertenezcan naturalmente a ciertas instituciones, ${ }^{4} \mathrm{ni}$ tampoco que exista un Derecho Natural al que la ley positiva debe conformarse. De lo anterior se sigue que ante el argumento expuesto hay un primer asunto a debatir, consistente en determinar si como sociedad aceptamos la existencia. autenticidad e imparcialidad de estas cosas que se dicen naturales y del Derecho Natural y si estamos dispuestos a que en nombre del Derecho Natural se legisle sobre temas que son de vital importancia para los ciudadanos. Y una segunda consideración, consistente en determinar si aún aceptando la existencia de características naturales a ciertas instituciones y del Derecho Natural y aplicando sus conceptos, es cierto que la indisolubilidad del matrimonio constituye uno de esos principios.

${ }^{4}$ Catharine A. Mackinnon. Toward a feminist theory of the state, 9, 46\& 54-59. (Harvard University Press 1989).

${ }^{5}$ Para una primerísima aproximacion al tema ver David Hume, Tratado de la naturaleza humana, ( Ed. Universidad Autónoma de Centro América. Clásicos de la democracia 1886). H.L.A. Hart, El concepto del derecho, ( Ed. Abeledo -Perrot. $2^{a}$ edición) Bertrand Russell, "Has religion made useful contributions to civilizations?", en Why $i$ am not a christian and other essays on religion and related subjects. 
A.- Acerca del Derecho Natural y legitimidad de un argumento basado en él.

El primer problema que encontramos con el argumento descrito es determinar si un argumento basado en la teoría del Derecho Natural poseería la legitimidad necesaria como para ser traducido a la ley positiva e impuesto al resto de la sociedad. No pretendemos discutir la existencia del Derecho Natural en este artículo, lo que, por lo demás, ha sido objeto de extensa discusión, pero creemos necesario hacer presente algunas observaciones al respecto antes de pasar a nuestro segundo punto de discusión.

A modo de una primerísima aproximación a lo que es el Derecho Natural podríamos decir que éste está conformado por: (i) ciertos principios básicos y valores universales que todo hombre maduro e inteligente podría apreciar como buenos (ii) un conjunto de requerimientos metodológicos de razón práctica que nos permiten distinguir entre actos que son razonables y actos que no lo son y formular (iii) un conjunto de normas morales. Estos principios no sólo están trazados por la filosofia moral, ética o por las conductas individuales sino también por filosofia política y jurídica, por la jurisprudencia, la política y por la vida del ciudadano. Su existencia justifica la acción de la autoridad en la comunidad y requiere que la autoridad sea ejercida habitualmente de conformidad a la norma juridica. Los principios de ley natural explican la fuerza obligatoria de la ley positiva, incluso cuando las leyes no son deducidas de los mismos y la atención a dichos principios justifica que ciertas leyes sean consideradas defectuosas si es que no se conforman a ellos. ${ }^{6}$

Santo Tomás de Aquino, uno de los mayores exponentes de la teoría del Derecho Natural, distingue entre los primeros principios o principios evidentes de ley natural (aquellos principios y valores universales a que nos referimos en el número (i) anterior) y preceptos secundarios, que son conclusiones muy próximas de aquellos principios. Los segundos, a diferencia de lo ocurre con los primeros principios, no son evidentes sino que deben ser deducidos a través del ejercicio de la razón practica. Dentro de los primeros principios, él contempla;

"El bien ha de hacerse y buscarse y el mal evitarse...

(....) De ahi que el orden de los preceptos de ley natural sea correlativo al orden de las inclinaciones naturales. Y así encontramos, ante todo una inclinación que le es común a todas las sustancias, consistente en que toda sustancia tiene por naturaleza conservar su propio ser. Y de acuerdo con esa inclinación pertenece a ley natural todo aquello que ayuda a la conservación de la vida humana e impide su destrucción.

En segundo lugar, encontramos en el hombre una inclinación hacia

${ }^{6}$ John Finnis. Natural law and natural rights, 23-25 (Oxford University Press. Ed. 1980). 
bienes más determinados, según la naturaleza que tiene en común con todos los demás animales, $\mathrm{y}$, al tenor de esta inclinación, se consideran de ley natural las cosas que la ley ha enseñado a todos los animales, tales como la conjunción de los sexos, la educación de los hijos y otras cosas semejantes.

En tercer lugar, hay una inclinación al bien correspondiente a la naturaleza racional, que es la suya propia, como por ejemplo, la inclinación natural a buscar la verdad acerca de Dios y a vivir en sociedad. Y según eso pertenece a la ley natural, todo lo que atañe a esta inclinación, como evitar la ignorancia, respetar a los conciudadanos y todo lo demás relacionado con eso."

Tal como está formulada, esta teoría presenta algunos problemas en cuanto a la legitimidad y en cuanto a su validez e imparcialidad. En efecto, hay un primer problema por lo cuestionable que es la existencia de primeros principios evidentes por sí mismos. En segundo lugar, también es controvertible el criterio de conformidad o contradicción con estos primeros principios que es la razón.

Respecto al primer problema, al de los principios evidentes por sí mismos. ¿Existen del todo? ¿Son tan evidentes como se clama? H.L.A. Hart entre muchos otros, objeta la concepción de un "Bien para el hombre" e indica que estos principios que se dice "evidentes", son a lo menos, complejos, debatibles y disputables. ${ }^{8}$ Por otra parte, quienes controvierten la existencia de estos primeros principios evidentes se preguntan si quienes sostienen la existencia de los mismos tienen derecho a exigir que se reconozcan como principios evidentes por todos. $^{9}$ y más aún. si pueden obligar a que dichos principios sean necesariamente recogidos como ley positiva de un país.

El segundo dilema lo constituye el criterio de conformidad con los principios evidentes, que los ius-naturalistas indican que es la razón práctica. Los detractores de esta teoría señalan que la razón no tiene el rol determinante que le adjudican los naturalistas, ni es imparcial. Para ellos. sería imposible desarrollar una teoría de Derecho Natural que fuera neutral. que no estuviera influenciada por determinadas maneras de pensar o creencias religiosas.

Dicho de otra manera, la teoría del Derecho Natural parte de la premisa que hay unos primeros principios evidentes, que serían de ley natural: contempla, asimismo, otros principios que, por su estrecha vinculación con los primeros principios evidentes, deben ser considerados de ley natural y expresados como Derecho Natural. En la determinación de los últimos, no sería la razón la que

${ }^{7}$ Tomás de Aquino, Suma teológica. Q. 94. Art. 2.

${ }^{8}$ Para mayor discusión acerca de la complejidad, debatibilidad y disputabilidad de estos principios ver: H.L.A Hart, The concept of law.

"Julius Stone, "Human Law and Human Justice", mencionado por Finnis en supra 6. en pag. 31 . 
jugaría un papel principal sino experiencias, creencias religiosas y morales. Agregan además que si fuera la razón el criterio de conformidad, ésta estaría viciada porque adolecería de parcialidad, ya que el razonar en sí mismo no es objetivo. ${ }^{10}$ David Hume, que es un ácido crítico de la teoría del Derecho Natural y en particular del rol que juega la razón en el proceso de determinación de sus principios es bastante claro en este sentido:

"En todo sistema de moralidad que hasta ahora he encontrado he notado siempre que el autor procede durante algún tiempo según el modo corriente de razonar, y establece la existencia de Dios mediante observaciones corrientes a los asuntos humanos, y de repente me veo sorprendido al hallar que en lugar de los enlaces usuales de las proposiciones es o no es, encuentro que ninguna proposición se haya entrelazada más que con debe o no debe. Este cambio es imperceptible, pero es, sin embargo, de gran consecuencia, pues como este debe o no debe expresa una nueva relación de afirmación, es necesaria que sea observada y explicada y al mismo tiempo debe darse una razón para que no sea completamente inconcebible, a saber; como esta nueva relación puede ser totalmente deducción de otras que son totalmente diferentes de ella, ya que los autores usan comúnmente esta precaución, debo aventurarme a recomendarla a los lectores, y estoy persuadido de que esta pequeña atención acabará con todos los sistemas corrientes de moralidad y nos permitirá ver que la distinción de vicio y virtud no se funda meramente en las relaciones de los objetos ni se percibe por la razón."'"

Los problemas esbozados nos hacen cuestionarnos qué es lo que efectivamente nuestra sociedad cree. Si cree efectivamente en la existencia de estos primeros principios evidentes o no, si cree que estos son efectivamente naturales o más bien son convenciones, puesto que si no existen o son convencionales son claramente modificables. Asimismo, nos hace preguntarnos acerca de la seguridad y objetividad del razonamiento para descubrir qué normas son efectivamente de Derecho Natural, y establecer si aún creyendo que existen propiedades naturales a determinadas instituciones, todo aquello que se indica como natural, lo es en verdad.

Habiendo dejado planteadas dichas inquietudes pasemos a nuestro segundo punto de discusión.

${ }^{10}$ Para una primera aproximación a la discusión ver: Richard Rorty "Solidarity on Objectivity" en Objectivity, relativism and truth, y Richard Delgado en "The inward truth in outsider jurisprudence" 34 William and Mary, L. Rev. (1993), en contra de la objetividad en el razonamiento y a favor de ella: Heidi Li Feldman. "Objectivity in Legal Judgement" 92 Mich. L. Rev. (1994) y Tomas Nagel en The last word. (1997)

" David Hume, Tratado de la naturaleza humana. p. 223 (Ed. Universidad Autónoma de Centro América. Clásicos de la democracia 1886). 
B.- Asumiendo que el Derecho Natural existe, ¿es la indisolubilidad del matrimonio uno de sus preceptos?

En segundo lugar es necesario establecer si aún reconociendo la existencia del Derecho Natural es cierto que la indisolubilidad del matrimonio es uno de los mandatos y si por tanto sería ilegítima una ley que admitiera la disolución del vínculo. Para tal efecto veremos primero si dicha conclusión se sigue necesariamente de los principios señalados por Santo Tomás de Aquino, y luego si ella podría desprenderse de interpretaciones más modernas y seculares del Derecho Natural.

Tal como se mencionó anteriormente, Santo Tomás establece cuáles son los primeros principios evidentes de ley natural y luego encarga a la razón práctica la tarea de descubrir los preceptos que de estos se derivan. Creemos encontrar en este proceso de raciocinio su mayor problema. Para Santo Tomás la virtud humana es análoga a la que se predica de cualquier otro espécimen de la naturaleza que se encuentre en buena forma, agregando que la virtud es lo que corresponde a la naturaleza humana, y que por el contrario el vicio es contra natura. Él une naturaleza y bondad, y, por ello, cuando analiza los preceptos a la luz de la razón práctica, no hace referencia a la naturaleza humana sino a la bondad humana ${ }^{12}$ que en él se confunden en una sola. Esto hace que en definitiva pase sin mayor explicación de lo que las cosas son a lo que las cosas deberían ser, perjudicando en alguna forma los fundamentos de sus tesis. Creemos que este problema se manifiesta cuando concluye que el matrimonio es indisoluble por naturaleza.

Cuando Santo Tomás establece que la indisolubilidad del matrimonio es un precepto de ley natural, lo hace porque indica que ella es necesaria para la consecución de los dos fines del matrimonio; tanto el fin primario que es la procreación de los hijos, como el secundario, que para él es la convivencia de los cónyuges. En esto hay un primer asunto discutible. El que estos sean los fines del matrimonio es enteramente debatible. No dudamos de que ellos pueden ser bienes para un matrimonio, pero ¿Son sus propiedades esenciales o sus fines? ¿No es caer en el problema a que nos referiamos en el párrafo anterior, cuando hablábamos de que Santo Tomás confunde la bondad con lo natural? Por otra parte, la enunciación de estos fines y su aceptación sin discusión constituye, como vimos, uno de los principales problemas que tiene la aplicación del Derecho Natural, que es que lo que resulta evidente para algunos no resulta evidente para todos.

Sigamos, en cuanto al fin primario, Santo Tomás afirma:

${ }^{12}$ Supra 6 en p. $35-36$. 
"Es pues natural que la solicitud del padre con su hijo se tenga hasta el final de su vida. Si, por consiguiente, la solicitud del padre por el hijo, causa aún en las aves la convivencia del macho y de hembra, el orden natural exige que en la especie humana cohabiten el padre y la madre". ${ }^{13}$

Si bien es cierto que parece razonable la primera hipótesis, esto es que la solicitud del padre por su hijo debiera durar por toda la vida, la conclusión de la indisolubilidad del matrimonio no se sigue de esta "solicitud". Y decimos que no se sigue por las siguientes razones:

(i) - No se da ningún argumento para establecer que es la solicitud del padre con el hijo la que causa la cohabitación del macho con la hembra.

(ii) - Tampoco se explica por que esta cohabitación deba ser para siempre, ni se acredita que en todas las aves o el resto de los animales la convivencia sea perpetua, aún cuando pudiera considerarse favorable para la educación de los hijos. ¿Que justificación tendría cuando los hijos ya no están siendo educados por sus padres?

(iii) - Aparece de la mera experiencia que el amor del padre por el hijo puede durar y de hecho dura aunque los padres no estén unidos, porque los padres quieren a sus hijos con independencia de sus cónyuges. Prueba de ello es. por ejemplo, que los viudos o viudas siguen queriendo a sus hijos. Entonces, ¿Por qué se exige la perpetuidad de la cohabitación para el bien de este amor filial?

Por otra parte las conclusiones de Santo Tomás cojean ante situaciones concretas: ¿Como podría justificarse esta propiedad de la indisolubilidad en matrimonios que no tienen hijos? ¿Cómo podría sostenerse que la indisolubilidad del matrimonio se debe por el bien de la prole, cuando éstos por ejemplo, ya no viven en la casa, o son adultos?

A mayor abundamiento, cabe indicar que si lo que estamos discutiendo es que el matrimonio es indisoluble, porque padre y madre deben cohabitar a perpetuidad por el bien de su hijos, esto no se consigue prohibiendo el divorcio sino prohibiendo las separaciones. El divorcio la mayoría de las veces sigue a una separación. El si efectivamente lo que se quiere es obligar a que padre y madre cohabiten por el resto de sus días por el bien de sus hijos, lo que debería hacerse es legislar prohibiendo las separaciones. Y creemos que sería dificil sino imposible sostener que el legislador pudiera o debiese prohibir las separaciones en virtud de los principios de ley natural para el cumplimiento de este fin primario a que nos hemos referido. Ello implicaría una clara y arbitraria intervención a la autonomía individual.

Santo Tomás señala que la indisolubilidad del matrimonio es necesaria para la obtención del fin secundario del matrimonio, que es la convivencia conyugal.

${ }^{13}$ Suma Contra Gentiles, L.3,a.CXXIII. 
La convivencia genera el amor de amistad, que es aquel por el cual se quiere el bien del otro, no por que sea un bien para uno, sino por el otro. En este sentido indica:

"La amistad, cuanto mayor es, tanto más es firme y duradera. Entre el varón y la mujer existe máxima amistad: pues se unen no sólo en el acto de la cópula carnal, que aún entre las bestias produce cierta suave sociedad. sino también para el consorcio de toda la vida del hogar, por donde en señal de esto, el hombre deja por la esposa incluso al padre y a la madre, como se dice en el génesis." ${ }^{14}$

Si bien es cierto que la convivencia favorece el bien de amistad, ello no implica que la única fórmula para su desarrollo sea la convivencia perpetua. Una cosa es que, como señala Santo Tomás, la convivencia sea favorable para este amor de amistad e incluso para esta máxima amistad y otra, que la convivencia deba durar para siempre. Es perfectamente posible que por el propio bien de cónyuges que no se avienen, que éstos no vivan juntos.

Por otro lado, cabe decir que el razonamiento de que el bien de amistad debe durar toda la vida porque entre hombre y mujer casados existe una máxima amistad, no se pone en la situación de que esta máxima amistad se acabe, como ocurre muchas veces en la práctica. No existiria justificación para mantener la convivencia cuando su premisa causal, esto es, el desarrollo de la amistad a la que nos hemos referido, no se puede lograr precisamente por haber desaparecido.

Es importante hacer notar que tampoco es el divorcio el que vulnera el fin de convivencia, sino la separación de los cónyuges. Por tanto, si hubiera una ley ilegítima en este sentido, ésta sería la que permite la separación, no la que admite el divorcio, que es un reconocimiento de una separación ya existente.

Pareciera ser pues, a la luz de lo señalado anteriormente, que las razones esgrimidas por Santo Tomas para defender la indisolubilidad del matrimonio no son todo lo fundamentadas o concluyentes que quisiéramos. Veamos si es que corrientes más modernas de Derecho Natural nos permiten encontrar buenas razones que nos convenzan de que la indisolubilidad del matrimonio es un precepto de Derecho Natural.

John Finnis, uno de los intelectuales vivos más influyentes en la teoría del Derecho Natural, contempla una serie de bienes básicos para el hombre, que son también sus derechos. Estos son: la vida, el conocimiento, el juego, la experiencia estética, la sociabilidad (amistad), la religión y la razón práctica. El indica que estos derechos son igualmente importantes y que ninguno de ellos debe primar por sobre los otros, pero considera de vital importancia a la razón práctica, ya que permite al hombre elegir entre las distintas acciones y estilos de

${ }^{14}$ Id. 
vida y formar su propio carácter, ${ }^{15}$ además de ser el medio para la dilucidación de las normas de Derecho Natural.

El correcto proceso de razonamiento, en el entender de Finnis, importa ciertos requisitos copulativos, a saber: armonía de propósitos, reconocimiento y respeto a los bienes básicos anteriormente mencionados, no privilegio de algunos bienes sobre otros ni favorecimiento de algunas personas o grupos, y eficiencia y autenticidad en la conformidad a la razón. Finnis distingue entre la razón práctica a nivel personal y a nivel comunitario. A nivel individual, la razón práctica permite al individuo su determinación personal y su participación en los bienes básicos. A nivel comunitario, el proceso de razonamiento permite la determinación de cuáles son los principios de Derecho Natural. Al pasar a este nivel agrega un nuevo requisito: el razonamiento no sólo debe ser realizado en conformidad a los parámetros señalados anteriormente, sino también como lo haría un observador independiente, esto es "quien puede contemplar a cada uno de los participantes en estas materias, con imparcialidad tanto afectiva como intelectual en relación a sus puntos de vista, estándares y principios de justicia." ${ }^{16}$

Tampoco se puede desprender que la indisolubilidad del matrimonio sea un principio de Derecho Natural de acuerdo a lo expuesto por John Finnis. Si bien es cierto que podría hacerse una relación entre el matrimonio y el bien de sociabilidad (que incluye a la amistad), esta relación no implica que la convivencia deba ser perpetua, ni implica que sea de ley natural que el matrimonio sea indisoluble o que, una ley de divorcio se ilegítima. Por otra parte, se podría incluso decir que la inexistencia o ilicitud del divorcio atentaría contra otro bien básico que es la razón práctica, por dos razones: la primera es que en el nivel individual de raciocinio se coartaría la libertad de autodeterminarse sin existir una razón lo suficientemente fuerte como para justificarlo; y la segunda, es que declarar la ilegitimidad de una ley de divorcio como un precepto de Derecho Natural no se compadecería con los preceptos de raciocinio nombrados en el párrafo anterior, puesto que implicaría; (i) arbitrariedad en la búsqueda de las normas, (ii) que se privilegiaran algunos bienes por sobre otros, por ejemplo amistad por sobre razón practica, o religión sobre razón práctica (iii) y restar autenticidad y eficacia en el raciocinio por que se estaría estableciendo la existencia de un principio careciendo de reales fundamentos para lo sustentarlo.

Entonces, la pregunta recurre. ¿Existe alguna otra razón que sea fuerte e imparcial, que pueda llevarnos de los primeros principios evidentes, o del bien de amistad individualizado por Finnis a sostener que el matrimonio es por su naturaleza indisoluble, y que una ley de divorcio atentaría contra dicha institución y sería contraria al Derecho Natural?

\footnotetext{
${ }^{15}$ Supra 6 pag. 88

${ }^{16}$ Supra 6 en pag. 108
} 
Varias razones han sido esgrimidas. Nuestro próximo propósito es analizarlas a la luz de la razón práctica imparcial en los términos de Finnis. La Iglesia Católica tiene sus razones, pero como veremos, parecen más bien referirse al sacramento del matrimonio. El estado de Chile, si es que tiene seriamente razones para sostener que el matrimonio es indisoluble por naturaleza, no sólo no las ha hecho valer, sino que ha permitido en la práctica que los matrimonios se disuelvan a través de los procesos de nulidad. En el resto del mundo occidental tampoco existen argumentos que permitan sostener que la indisolubilidad del matrimonio es un precepto de Derecho Natural ¿Qué pasa entonces, podemos encontrar esta razón imparcial que nos interesa o no?

Tradicionalmente la Iglesia Católica, siguiendo lo sostenido por San Agustín, ha manifestado que el matrimonio tiene tres fines o propiedades naturales: bonum proli, bonum fidei, y bonum sacramenti. No podria excluirse del matrimonio la copulación procreativa, porque ello iría en contra del bonum proli; no podría uno de los cónyuges reservarse el derecho a tener relaciones sexuales con una tercera persona, porque ello violaría el bonum fidei y: finalmente, no podrian las partes reservarse el derecho a poner término al matrimonio si una dificultad apareciera, porque ello sería contrario al bonum sacramenti.

Primeramente, es interesante notar la denominación que utiliza la Iglesia para referirse a la indisolubilidad del matrimonio, bonum sacramenti. En otras palabras, la indisolubilidad es un bien en razón del sacramento. Esto cobra especial importancia ya que los sacramentos son instituciones esencialmente religiosas, son signos y medios por los cuales se expresa y se refuerza la fe, se adora a Dios. y en los que la persona se santifica. ${ }^{17}$ En la celebración del sacramento del matrimonio, el sacerdote sentencia: "Lo que Dios ha unido, no lo separe el hombre". Esta sentencia hace perfectamente entendible y saludable que el sacramento del matrimonio sea perpetuo, pero ello no implica que se pueda traspasar dicha propiedad de perpetuidad al contrato secular de matrimonio, porque justamente los matrimonios civiles no son "bendecidos" por Dios.

El derecho canónico se refiere extensamente a la indisolubilidad del matrimonio, sin embargo estas normas no tienen mayor alcance que regular los sacramentos matrimoniales entre católicos. Es interesante mencionar que aún en los cánones religiosos la indisolubilidad del matrimonio tiene excepciones. La existencia de éstas reafirman la idea que hemos venido sosteniendo hasta en este artículo de que la indisolubilidad no es algo esencial del matrimonio, ya que lo esencial es justamente aquello de que no puede nunca prescindirse.

Por ejemplo, el Canon 1143 establece el Privilegio Paulino. En virtud del mismo, el matrimonio entre dos no bautizados puede ser disuelto en favor de la

${ }^{17}$ Código De Derecho Canónico. Canon 840. 
parte que recibe el bautismo. Esta es una clara excepción a la indisolubilidad. A través de él, de alguna manera la Iglesia reconoce que un matrimonio entre no bautizados puede ser disuelto, de lo que sigue que no necesariamente y para todos el matrimonio es indisoluble sino sólo para los bautizados, porque así lo dispone el Código canónico en atención a la especial peculiaridad del sacramento.

Existe también otro argumento interesante que refrenda la idea de que la indisolubilidad no es esencial al "contrato" de matrimonio, ni aún en el derecho canónico. El matrimonio es tanto para la ley religiosa como para la ley civil, un contrato (para la primera no es sólo un contrato sino también un sacramento). En el derecho civil los elementos esenciales de los contratos deben estar siempre presentes, de no ser así. el contrato es nulo o degenera en otro distinto. Así, por ejemplo, en una compraventa la cosa y el precio son elementos esenciales del contrato. Si una de las partes cree comprar un candelabro y la otra esta vendiendo otra cosa distinta, o si uno cree que va a recibir un precio por su mercadería en circunstancias que la otra parte no está dispuesta a pagar por su adquisición, el contrato o será un contrato de compraventa anulable (o inexistente) o degenerará en otro distinto. Pues bien, el Canon 1099 establece que el error concerniente a la indisolubilidad del matrimonio no vicia el matrimonio. Si la indisolubilidad fuera un elemento esencial del contrato de matrimonio, un error acerca de él viciaría el contrato; a contrario sensu, si un error acerca de la indisolubilidad del matrimonio no invalida el contrato, es porque no es realmente un elemento esencial del mismo. ${ }^{18}$

La Iglesia Católica tiene, en verdad, razones poderosas para exigir la indisolubilidad del matrimonio. Sin embargo, no encontramos razones igualmente poderosas que nos convenzan de su natural indisolubilidad cuando tratamos el matrimonio desde la perspectiva de contrato secular.

En segundo lugar, ¿Tiene el estado razones poderosas para sostener que el matrimonio una institución naturalmente indisoluble y por tanto prohibir su disolución?

Los argumentos que se han esgrimido han ido generalmente de la mano de la noción de que el matrimonio es una institución indisoluble por naturaleza. Sin embargo, en la práctica, el estado ha ignorado los miles de matrimonios que se disuelven a través del proceso de nulidad, de lo que se deduce que de alguna manera, no hay un verdadero convencimiento de que el matrimonio sea indisoluble per se. ¿Existe pues alguna otra razón, esgrimida por un observador independiente, como propone Finnis, que permita convencernos de que la indisolubilidad del matrimonio es una principio de Derecho Natural?

En el resto de los paises de occidente, no encontramos ningún país que considere el matrimonio como una institución indisoluble por naturaleza y que

${ }^{18}$ Todo lo anterior no obsta a que sea un elemento esencial al sacramento. 
asi lo tenga expresado en la ley positiva. En todos ellos el divorcio está legalizado. La razón de esto es que se considera que ponerle término al matrimonio es del tipo de decisiones que debe ser tomada por los ciudadanos, y que los efectos de dicha decisión sólo deben ser regulados en lo que afectaren a terceros. ${ }^{19}$ También existen razones de orden práctico, pues se considera más nocivo mantener familias en situación irregular a permitir un divorcio que, aunque nunca es una situación grata para la familia, la afecta menos que pretender artificialmente la indisolubilidad de un matrimonio en efecto "disuelto" o roto.

\section{III.- Conclusiones, ¿Qué irá a suceder?}

Está claro que el actual sistema de nulidad no puede mantenerse puesto que está dañando a nuestra sociedad y a nuestro sistema legal. Por otro lado esta también claro que no todos los chilenos creen en la indisolubilidad de sus matrimonios, y de hecho en la práctica buscan la manera de "disolverlos" cuando ellos fracasan. En este contexto se hace necesario buscar una solución al problema. Una de las alternativas es legislar permitiendo el divorcio. Los argumentos que se han dado para impedir una legislación en este sentido son varios. Nosotros hemos querido en este artículo referirnos especialmente a uno: aquel que postula que el matrimonio es naturalmente indisoluble, que su indisolubilidad es de Derecho Natural y que por tanto una ley positiva que permitiere el divorcio sería ilegitima e injusta.

Nos parece que los fundamentos para sostener lo anterior no son lo suficientemente convincentes. No sólo no está clara la legitimidad de un argumento basado en el Derecho Natural en una sociedad que no necesariamente acepta la existencia del mismo, sino que tampoco aparece patente que una ley de divorcio sea ilegítima o contraria al Derecho Natural, toda vez que dicha conclusión no se desprende necesariamente de los principios de Santo Tomás; ni se conforma a lo postulado por otros ius naturalistas. Aún más podría aducirse que una ley que permitiera el divorcio no sólo no sería contraria a la ley natural, sino que el no tenerla incluso vulneraria a lo menos uno de los bienes considerados como bienes básicos del hombre, el de la razón práctica, puesto que sin suficiente justificación impediría la autodeterminación de las personas.

Asi las cosas, pareciera en nuestra opinión que son consideraciones religiosas las que están liderando este debate, disfrazadas de una capa de neutralidad que, en verdad, no tienen. Se argumenta que la indisolubilidad del matrimonio prescindiría de toda consideración religiosa, lo que sería, la mayor

${ }^{19}$ Para data, ver Mary Ann Glendon, en "Abortion and divorce in western law", p, 68.9, (Harvard University Press 1987). A la época de publicación de este libro el único pais europeo que prohibía el divorcio era Irlanda, pais que lo aprobó el año 1996. 
virtud de la teoria y, sin embargo, desgraciadamente no encontramos más argumentos que religiosos. Conviene aclarar, eso si, que el problema no es la existencia de argumentos religiosos per se: por el contrario, es sumamente legítimo que la iglesia se autorregule. El problema es pretender que sean preceptos religiosos los que regulen la vida civil de los ciudadanos.

Estamos persuadidos de que las consideraciones religiosas o sus mandamientos no deben ser la base para la acción del estado o para la dictación de leyes. Por el contrario, parece razonable sostener, como lo hizo la misma Iglesia Católica en el Concilio Vaticano II, que la búsqueda de la verdad religiosa es un asunto personal que requiere una decisión consciente y no de un mandamiento que pueda ser impuesto o que corresponda imponer a la autoridad civil. $^{20}$

Nuestra Constitución reconoce la separación entre Iglesia y Estado. Existe, además, cierto consenso en la conveniencia de que ambas instituciones se mantengan en las esferas que les corresponden: la pública y la privada-espiritual, respectivamente. La cuestión que queda por contestar es si vamos a tener la voluntad para hacer efectivo el mandato constitucional y mantener separadas las instituciones como constitucionalmente corresponde, o vamos a permitir que explicaciones o argumentaciones religiosas dominen la vida civil.

${ }^{20}$ John Locke, A letter concerning toleration. p. 26-28. (Hactkett Publishing Company 1983). Traducción Libre. 